\title{
Segmentation of Multiple Sclerosis Lesions in Brain MR Images Using Spatially Constrained Possibilistic Fuzzy C-Means Classification
}

\author{
Hassan Khotanlou; Mahlagha Afrasiabi \\ H. Khotanlou and M. Afrasiabi are with Computer Engineering Dept., Bu-Ali Sina Univ., Hamedan, Iran, \\ email: \{khotanlou, m.afrasiabi\}@basu.ac.ir
}

\begin{abstract}
This paper introduces a novel methodology for the segmentation of brain MS lesions in MRI volumes using a new clustering algorithm named SCPFCM. SCPFCM uses membership, typicality and spatial information to cluster each voxel. The proposed method relies on an initial segmentation of MS lesions in T1-w and T2-w images by applying SCPFCM algorithm, and the T1 image is then used as a mask and is compared with T2 image. The proposed method was applied to 10 clinical MRI datasets. The results obtained on different types of lesions have been evaluated by comparison with manual segmentations.
\end{abstract}

\section{KEYWORDS}

Fuzzy classification, spatial information, brain imaging, multiple sclerosis lesions

\section{INTRODUCTION}

Multiple sclerosis (MS) is a progressive neurological disorder, which is caused by structural damages of axons and their myelin sheathes in the central nervous system. MS lesions present temporal changes in shape, location, and area between patients, and thus it is necessary for radiologists to accurately detect and evaluate MS lesions [1]. However, the accurate assessment of each lesion in magnetic resonance (MR) images would be a demanding and time-consuming task, and also a manual measurement could be subjective and have poor reproducibility. Therefore, a number of semi-automated or automated methods have been proposed for identifying and/or segmenting MS lesions based on two or three-dimensional (3-D) MR images.

By Khayati et al. [2] an approach was proposed for fully automated segmentation of MS lesions in fluid attenuated inversion recovery (FLAIR) MR images. The proposed approach, based on a Bayesian classifier, utilizes the adaptive mixtures method (AMM) and Markov random field (MRF) model to obtain and upgrade the class conditional probability density function (CCPDF) and $a$ priori probability of each class. A mean value equals to 0.75, was obtained for Similarity Index (SI).

Anbeek et al. [3] proposed a novel automatic approach for segmentation of white matter lesions in MR images of brain. The introduced algorithm uses different information, including voxel intensity and the spatial information, to classify voxels by a K-nearest neighbor (KNN) classifier. This technique assigns a probability to each voxel for being part of white matter lesion. The SI is then used for determination of an optimal threshold on the probability map to segment the images.

Lorenzo et al. [4] suggested an approach that uses the information from the proton density (PD), T2-weighted and FLAIR images. This strategy involved CSF and lesion classification using the Parzen window classifier. Image preprocessing, morphological operations and ratio maps of PD and T2 weighted images are used for minimizing false positives. Contextual information is exploited for minimizing the false negative lesion classifications using hidden Markov random field and expectation maximization (HMRF-EM) algorithms. Lesions are delineated using fuzzy connectivity.

Prastawa et al. [5] presented a novel, fully automatic, segmentation method for MS lesions in brain MRI that combines outlier detection and region partitioning. The method uses an atlas of healthy subjects and detected lesions as outliers, without requiring the use of training data with segmented lesions.

Ardizzone et al. [6] introduced a novel approach to the detection of MS lesions in T2- and PD-weighted MR images. The core of the proposed method is the use of the two channels fuzzy c-means (FCM) segmentation of data, where the classical FCM approach runs, at first, on the two separate spectra. Then, the one dimensional distributions of the cluster centers obtained by FCM are composed in the two-dimensional one, which is a-priori imposed to the two-spectrum segmentation procedure.

Admasu et al. [7] suggested a method that combines the strengths of two existing techniques: fuzzy connectedness and artificial neural networks. From the input MR brain image, the fuzzy connectedness algorithm was used to 
extract segments which were parts of Cerebrospinal Fluid (CSF), White Matter (WM) or Gray Matter (GM). Segments of the MR image which were not extracted as part of CSF, WM or GM were processed morphologically, and features were computed for each of them. Then these computed features were fed to a trained artificial neural network, which decided whether a segment was a part of a lesion or not.

Admiraal-Behloul et al. [8] suggested a fully automatic segmentation method for quantifying white matter hyper intensity in a large clinical trial on elderly patients. The introduced algorithm combines information from three different MR images including PD, T2-w and FLAIR. It uses FCM algorithm for clustering process. The approach demonstrated very high volumetric and spatial agreement with expert delineation.

In the most of the proposed methods, spatial information have not been considered for segmentation and they fail in the presence of noise and week border of MS lesions. In this paper we propose a new method that is based on a modified possiblistic fuzzy c-mean. This method uses membership, typicality and spatial information to extract MS lesions.

\section{RELATED WORKS: FUZZY CLASSIFICATION USING MEMBERSHIP, TYPICALITY AND SPATIAL INFORMATION}

As usual in fuzzy clustering, we consider the problem of partitioning an unlabeled data set $X=\left\{x_{1}, x_{2} \ldots, x_{n}\right\} \subset R^{p}$ into $c$ classes $(1<c<n)$. A c-partition of $X$ is a set of ( $c n)$ values $u_{i k}$ that can be represented as a $(c \times n)$ matrix $U=\left\{u_{i k}\right\}$ [9]. The value $u_{i k}$ denotes the membership degree of sample $x_{k}$ to class $i$.

One of the most widely used clustering methods is the FCM algorithm. The FCM algorithm assigns memberships to $x_{k}$ which are related to the relative distance of $x_{k}$ to the $c$ points prototypes $V=\left\{v_{i}\right\}$ that are class centers in the FCM. It minimizes an objective function under a normalization constraint of $u_{i k}$.

The FCM algorithm has some drawbacks that have limited its application. The main one is that the membership functions are not decreasing with respect to the distance to the class center. To overcome this problem, a new clustering method named possibilistic c-mean (PCM) was proposed by [10].

In this algorithm the objective function is modified and the normalization constraint, $\quad \sum_{i=1}^{c} \boldsymbol{u}_{i k}=1, \forall k=1$ is not considered. The authors named the value $\mathrm{u}_{\mathrm{ik}}$ as typicality (typicality of $x_{k}$ relative to cluster $i$ ). In fact each row of $U$ is a possibility distribution over $X$. However this algorithm is sensitive to initialization and sometimes coincident clusters will occur. In addition it is sensitive to the additional parameters in this model.

To address the problems of FCM and PCM a new fuzzy possibilistic c-mean (FPCM) algorithm was proposed in [11] by combining these two algorithms. In data classification, both membership and typicality are useful for data structures interpretation and FPCM computes these two factors simultaneously.

FPCM solves the noise sensitivity defect of FCM and overcomes the problem of coincident clusters of PCM. The objective function of FPCM is written as:

$$
J_{m, \eta}(U, T, V ; X)=\sum_{i=1}^{c} \sum_{k=1}^{n}\left(u_{i k}^{m}+t_{i k}^{n}\right) D_{i k}
$$

where $m>1 \quad \bullet \eta>1 \quad \cdot 0 \leq u_{i k} \leq 1 \quad \cdot 0 \leq t_{i k} \leq 1 \quad$ ' $\sum_{i=1}^{c} u_{i k}=1, \forall k$ $\sum_{i=1}^{c} t_{i k}=1, \forall i \quad D_{i k}=\left\|x_{k}-v_{i}\right\|^{2} \quad(\|$.$\| \quad is any inner$ product norm). Here $T=\left\{u_{i k}\right\}$ is the typicality matrix.

Although FPCM is less prone to the problems of FCM and PCM, in the case of large data sets this algorithm does not work properly (it operates such as FCM), because FPCM normalizes the possibility values, so that the sum of typicality of all data points in each row of $U$ is one. Hence the typicality values are very small in large data sets.

A new algorithm for data clustering was proposed in [12], named possibilistic fuzzy c-means (PFCM). In this algorithm the constraint of the typicality values $\left(\sum_{i=1}^{c} t_{i k}=1, \forall i\right.$ )has been relaxed to overcome the problem of FPCM. The objective function of PFCM is written as:

$J_{m, \eta}(U, T, V ; X)=\sum_{i=1}^{c} \sum_{k=1}^{n}\left(a u_{i k}^{m}+b t_{i k}^{n}\right) D_{i k}+\sum_{i=1}^{c} \gamma_{i} \sum_{k=1}^{n}\left(1-t_{i k}\right)^{n}$

Where $\sum_{i=1}^{c} u_{i k}=1, \forall k \quad$ ، $0 \leq u_{i k}, t_{i k} \leq 1$ ، $a>0$ ، $b>0 \quad \gamma_{i}>0 \quad m>1$ are user defined constants. The relative importance of fuzzy membership $u_{i k}$ (as in FCM) and typicality $t_{i k}$ (as in PCM) in the objective function are defined by the constants $a$ and $b$. If $a=1, b=0$ and $\gamma_{i}=0, \forall i$, PFCM reduces to FCM and if $a=0$ and $b=1$ , it reduces to PCM. In [3] the following equation is suggested to compute $\gamma_{i}$ :

$\gamma_{i}=K \frac{\sum_{k=1}^{n} D_{i k}}{\sum_{k=1}^{n} u_{i k}^{m}}, K>1$

The PFCM algorithm overcomes the problems of PCM and FCM and functions properly on large data sets. It can easily be seen from equation (2) that the objective function of PFCM 
does not take into account any spatial information. Hence, it is sensitive to noise and intensity inhomogeneity, and its application for real MR image classification is very limited.

Recently, approaches have been proposed by modifying the objective function to increase the robustness of FCM to noise. Here we use a new algorithm (modified PFCM $(\mathrm{SCPFCM})$ )[13] which itself uses both the information of voxels and their neighborhoods (inspired from Markov Random Fields (MRF)), membership and typicality for classification.

The objective function of SCPFCM is written as:

$$
J_{m, \eta}(U, T, V ; X)=\sum_{i=1}^{c} \sum_{k=1}^{n}\left(a u_{i k}^{m}+b t_{i k}^{n}\right) D_{i k}+\sum_{i=1}^{c} \gamma_{i} \sum_{k=1}^{n}\left(1-t_{i k}\right)^{n}+\beta \sum_{i=1}^{c} \sum_{k=1}^{n}\left(a u_{i k}^{m}+b t_{i k}^{n}\right) S_{i k}
$$

Here $S_{i k}=\sum_{w=1}^{n_{w}}\left\|x_{w}-v_{i}\right\|^{2}$ where $x_{w}$ is a neighbor pixel/voxel of $x_{k}$ in a window around $x_{k}$ and $n_{w}$ is the number of neighbors in this window.

The relative importance of the added term (neighborhood effect) is controlled by $\beta$ which can be written as $\left.\frac{\alpha}{n_{w}}\right)$. If $m>1$ and $\eta>1$ then the objective function will be minimized for :

$u_{i k}=\sum_{j=1}^{c}\left(\frac{D_{i k}+\beta S_{i k}}{D_{j k}+\beta S_{j k}}\right)^{\frac{1}{1-m}}, 1 \leq i \leq c, 1 \leq k \leq n$

$t_{i k}=\frac{1}{1+\left(\frac{b}{\gamma_{i}} D_{i k}+\beta S_{i k}\right)^{1 /(\eta-1)}}, 1 \leq i \leq c, 1 \leq k \leq n$

$v_{i}=\frac{\sum_{k=1}^{n}\left(a u_{i k}^{m}+b t_{i k}^{n}\right)\left(x_{k}+\beta R_{k}\right)}{(1+\alpha) \sum_{k=1}^{n}\left(a u_{i k}^{m}+b t_{i k}^{n)}\right.}, 1 \leq i \leq c$

In summary, the SCPFCM algorithm can be written as:

(1) Select initial prototypes $V=\left\{v_{i}\right\}_{i=1}^{c}$

(2) Update the membership matrix using Equation (5).

(3) Update $\Gamma=\left\{\gamma_{i}\right\}_{i=1}^{c}$ using Equation (3).

(4) Update the typicality matrix using Equation (6).

(5) Update the prototypes using Equation (7).

(6) Repeat 2-5 until termination. The termination criterion is as follows:
$\left\|V_{\text {new }}-V_{\text {old }}\right\|<\varepsilon$

where $\|$.$\| is the Euclidean distance norm and \varepsilon$ is a small number, to be set by the user.

\section{Segmentation Algorithm}

The automated MS lesions segmentation method that we have developed is composed of two phases: initialization and refinement, as shown in Figure 1.

T1-w and T2-w images are first being segmented using SCPFCM algorithm, and then surrounding area of brain are separated in the two images with use of morphology functions[14]. After that remained parts of image T1-w is being used as a mask and remained parts of image T2-w is compared with this mask to extract MS lesions. Figure 2 shows the segmentation process on a flowchart.

MR images

(T1 and T2)

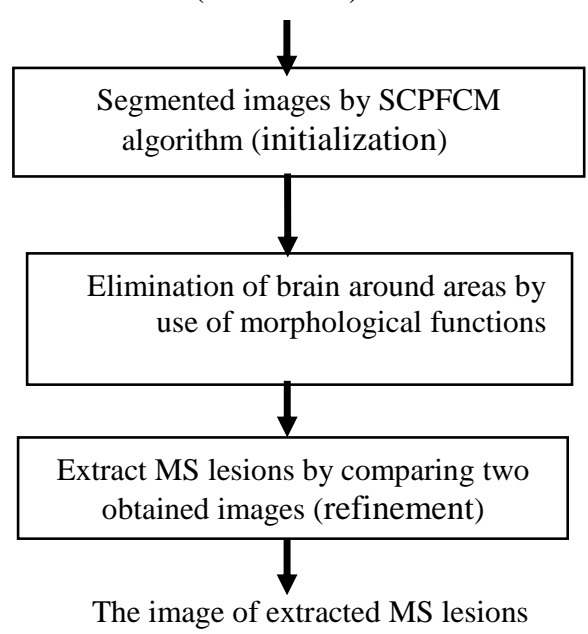

Figure 1: Block diagram of the proposed approach for fully automatic segmentation of MS lesions.

\section{Evaluation}

\section{A. MR imaging}

We applied the suggested method on two types of images 1) real MR images of 10 patients taken from Imam Khomeini hospital. Images were selected in this study according to the revised Mc Donald criteria 2005 [15]. The resolution and the size of each 3D voxel is $256 \times 256 \times 25$ and $0.97 \times 0.97 \times 4 \mathrm{~mm}^{3}$ respectively. The location and the size of lesion are different in these 10 patients. To compare the segmentation of lesions for patients with different lesion volumes $(\mathrm{LV})$, three patient categories, small $(L V<4 c c)$, moderate $(4 c c<L V<18 c c)$, and large lesion $(L V>18 c c)$ load, in our selected slices, were composed [2]. 

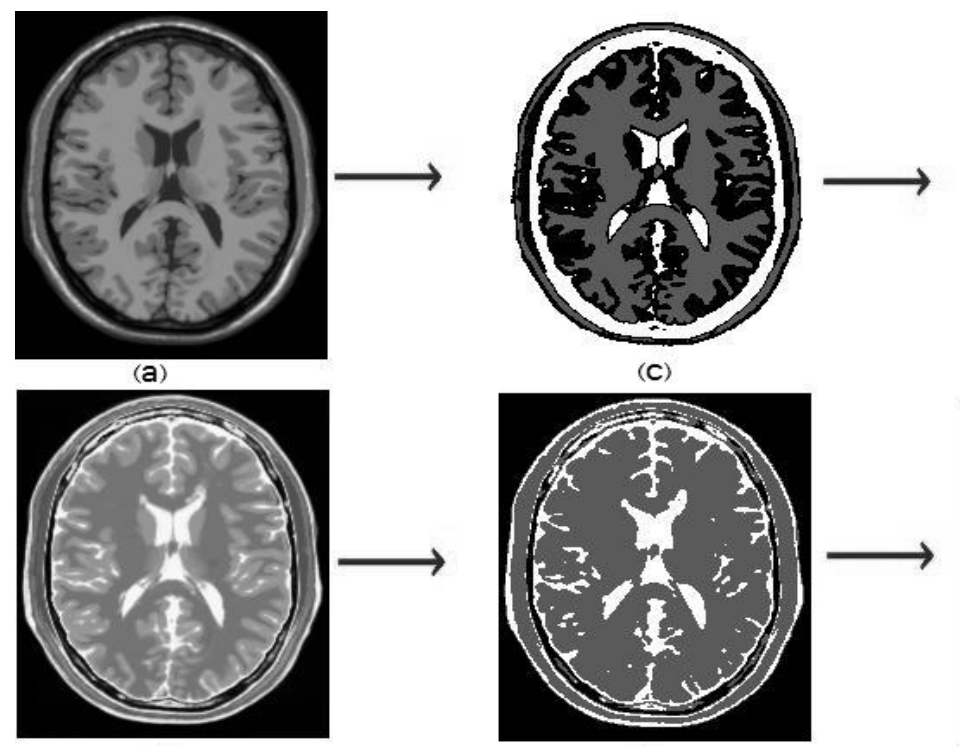

(b)

(d)

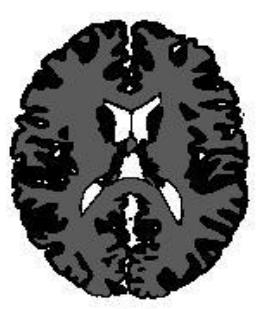

(e)

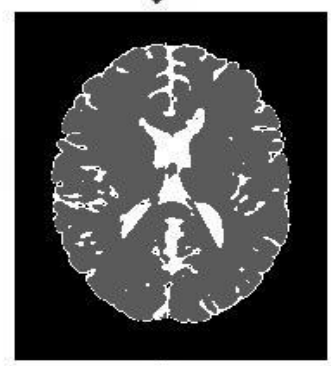

(f)

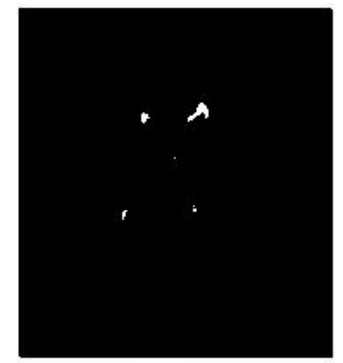

(g)

Figure 2: Segmentation of MS lesions in simulated image a) Simulated T1-w image. b) Simulated T2-w image. c) Clustering of T1-w image using SCPFCM d) Clustering of T2-w image using SCPFCM e,f) Extraction of brain. g) Extraction of MS lesions.

Accordingly, 4 out of 10 reviewed patients have small lesion, 4 have medium lesion and 2 have large lesion. 2) 3D simulated MR data with different levels of Gaussian noise [16]. The volume dimension is $256 \times 256 \times 25$ and the voxel size is $1 \times 1 \times 1 \mathrm{~mm}^{3}$.

\section{B. Evaluation}

Results of the lesions segmentation based on the proposed method are compared with the gold standard. The similarity index (SI) as illustrated in equation 9 [13], overlap fraction (OF) as shown in equation 10 and extra fraction (EF) as represented in equation 11 are calculated for the dataset images. The SI is a criterion for the correctly classified lesion area relative to the total areas of lesion, in both the gold standard and in the segmented image.

$$
\begin{aligned}
& S I=\frac{2 T P}{2 T P+F P+F N} \\
& O F=\frac{T P}{T P+F N} \\
& E F=\frac{F P}{T P+F N}
\end{aligned}
$$

In the equations 9-11, TP stands for true positive voxels, FP for false positive voxels, and FN for false negative voxels. SI and $\mathrm{OF}$ for a good segmentation should be close to 1 and EF should be close to 0 .

\section{Results}

The classifications results into 4 classes (background, CSF, WM, GM) obtained by FCM and SCPFCM on a noiseless simulated image and extracted MS lesions are shown in Figures 3 and 4. The results of extracted MS lesions by FCM and SCPFCM on this image with different levels of Gaussina noises are shown in Figure 5. Figure 6 shows MS segmentation result in a real MR image.

These results show that the proposed method removes the noises and classifies the voxels correctly. Setting the parameters of $\operatorname{SCPFCM}(a ; b ; \beta)$ with proper values is important. The parameter $\beta$ controls the neighborhood effect and it should be high enough for very noisy images. A small value of $\beta$ converts the SCPFCM algorithm to PFCM algorithm. Here we obtained the best results with $\beta=0.2$.

The parameter $a$ controls the effect of membership and the parameter $b$ specifies the effect of typicality. Setting these parameters depends on the type of images and the number of classes. Setting $b$ with a high value compared to $a$ causes the centroid be more influenced by the typicality values than the membership values and a coincidence problem may occur.

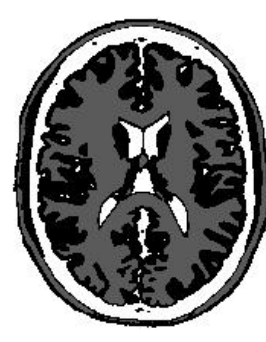

(a)

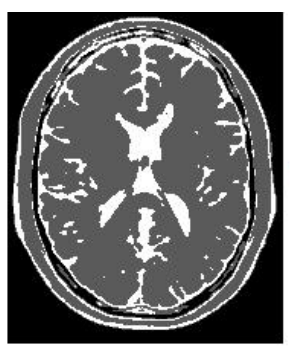

(b)

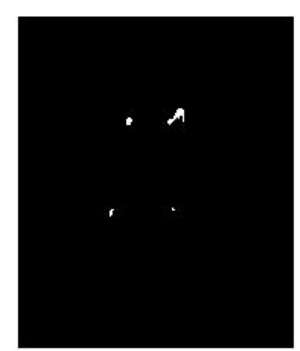

(C)
Figure 3: (a) Classification of T1-w image with FCM algorithm. (b) Classification of 21-w image with FCM algorithm. (c) Segmentation of MS lesions 


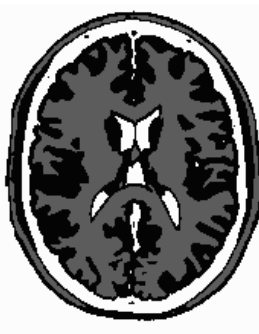

(a)

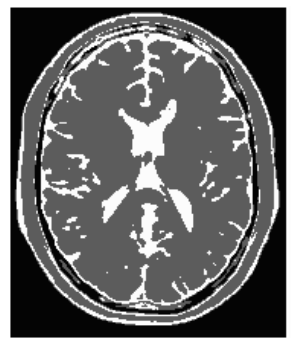

(b)

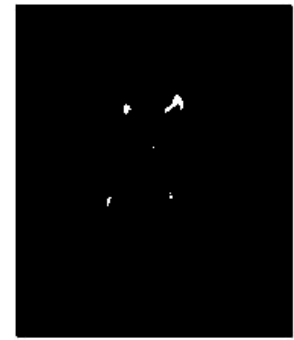

(C)
Figure 4: (a) Classification of T1-w image with SCPFCM algorithm (b) Clasification of T2-w image with SCPFCM algorithm (c) Segmentation of MS lesions

Conversely using a high value of $a$ compared to $b$ causes the centroids be more influenced by membership values and it may generate the FCM problems (for the parameters $m$ and $\eta$ similar effects can also be obtained).

\section{Quantitative Results and Discussion}

The quantitative results obtained with different algorithms are provided in Table 1 and Table 2 for five simulated images with different levels of Gaussian noise. As seen in Table 1 and Table 2 when the noise is low, the difference between the classification accuracies of the algorithms is low. When the noise increases, the difference between classification accuracies is increased, and the SCPFCM performs better than the other algorithms. For example the difference between the SI of FCM and SCPFCM in the image with $3 \%$ noise is about 9 units, while for the image with $7 \%$ noise, it is about 44 .

We compared the results of MS segmentation with three other methods in Table 4. It is reminded that these researchers has used manual segmentation for evaluation of their methods. We, too, used manual segmentation for evaluation. Therefore comparison of our method with these methods is reasonable. As it is seen in Table 4, the MS lesion segmentation algorithm used in this paper improves $7 \%, 7 \%, 2 \%$, the results reported in $[8,3,2]$. As shown in Table 4, this method works better than the three other ways based on lesion size classifying. For small and medium size lesions, it works completely better. For large lesions, it is almost like previous methods. The most important lesions to recognition are the tiny ones in which edges are not clear that the SCPFCM method classifies well. Although the method [3] has high accuracy in tiny lesions does not have that accuracy and is not considered as a good method.

Table1. Similarity index which have been obtained from FCM, FPCM, PFCM, and SCPFCM algorithms in simulated MR images .

\begin{tabular}{llllll}
\hline Algorithm & $0 \%$ & $1 \%$ & $3 \%$ & $5 \%$ & $7 \%$ \\
\hline FCM & 80.56 & 79.23 & 69.92 & 41.58 & 16.29 \\
FPCM & 81.84 & 79.65 & 75.17 & 69.11 & 51.67 \\
PFCM & 81.97 & 79.69 & 76.04 & 70.35 & 56.42 \\
SCPFCM & 89.02 & 85.11 & 78.95 & 73.44 & 60.11 \\
\hline
\end{tabular}

Table2. Overlap fraction which have been obtained from FCM, FPCM, PFCM, and SCPFCM algorithms in simulated MR images.

\begin{tabular}{llllll}
\hline Algorithm & $0 \%$ & $1 \%$ & $3 \%$ & $5 \%$ & $7 \%$ \\
\hline FCM & 91.24 & 89.9 & 83.74 & 32.66 & 9.69 \\
FPCM & 91.65 & 91.22 & 88.86 & 80.48 & 47.12 \\
PFCM & 92.07 & 91.32 & 88.90 & 81.24 & 53.75 \\
SCPFCM & 95.85 & 93.21 & 90.44 & 85.34 & 70.22 \\
\hline
\end{tabular}

Table 3. Average similarity index criteria of 10 real MR images which have been obtained from FCM, FPCM, PFCM, and SCPFCM algorithms.

\begin{tabular}{llll}
\hline Algorithm & SI & OF & EF \\
\hline FCM & 71.6 & 62.4 & 14.2 \\
FPCM & 75.9 & 69.9 & 13.9 \\
PFCM & 78.5 & 72.6 & 14.1 \\
SCPFCM & 82.6 & 77.1 & 10.3 \\
\hline
\end{tabular}
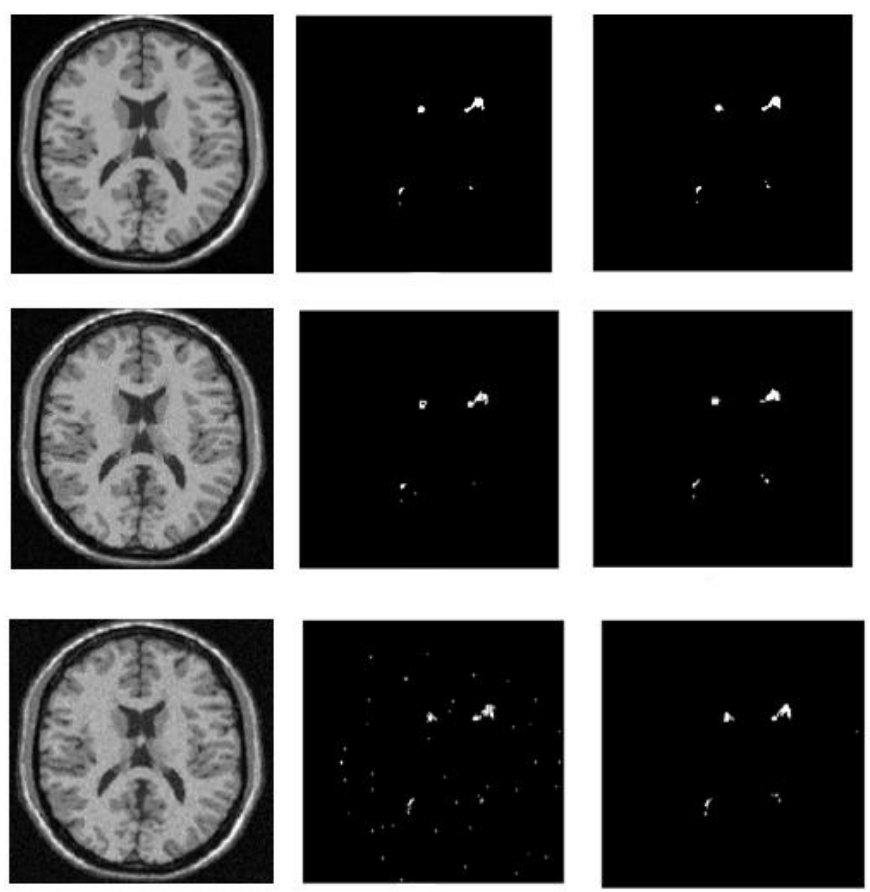

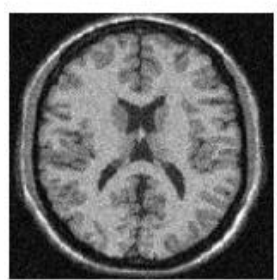

(a)

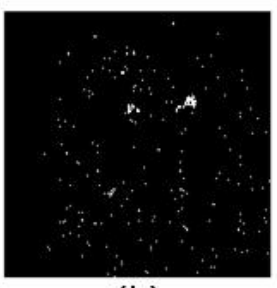

(b)

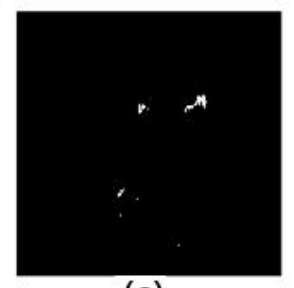

(c)
Figure 5: Segmentation of MS lesions in simulated images. (a) Original T1-w images wit 1\%,3\%, 5\%, $7 \%$ noises respectively from top to buttom. (b) Extraction of MS lesions using FCM algorithm. (c) Extraction of MS lesions using SCPFCM algorithm. 

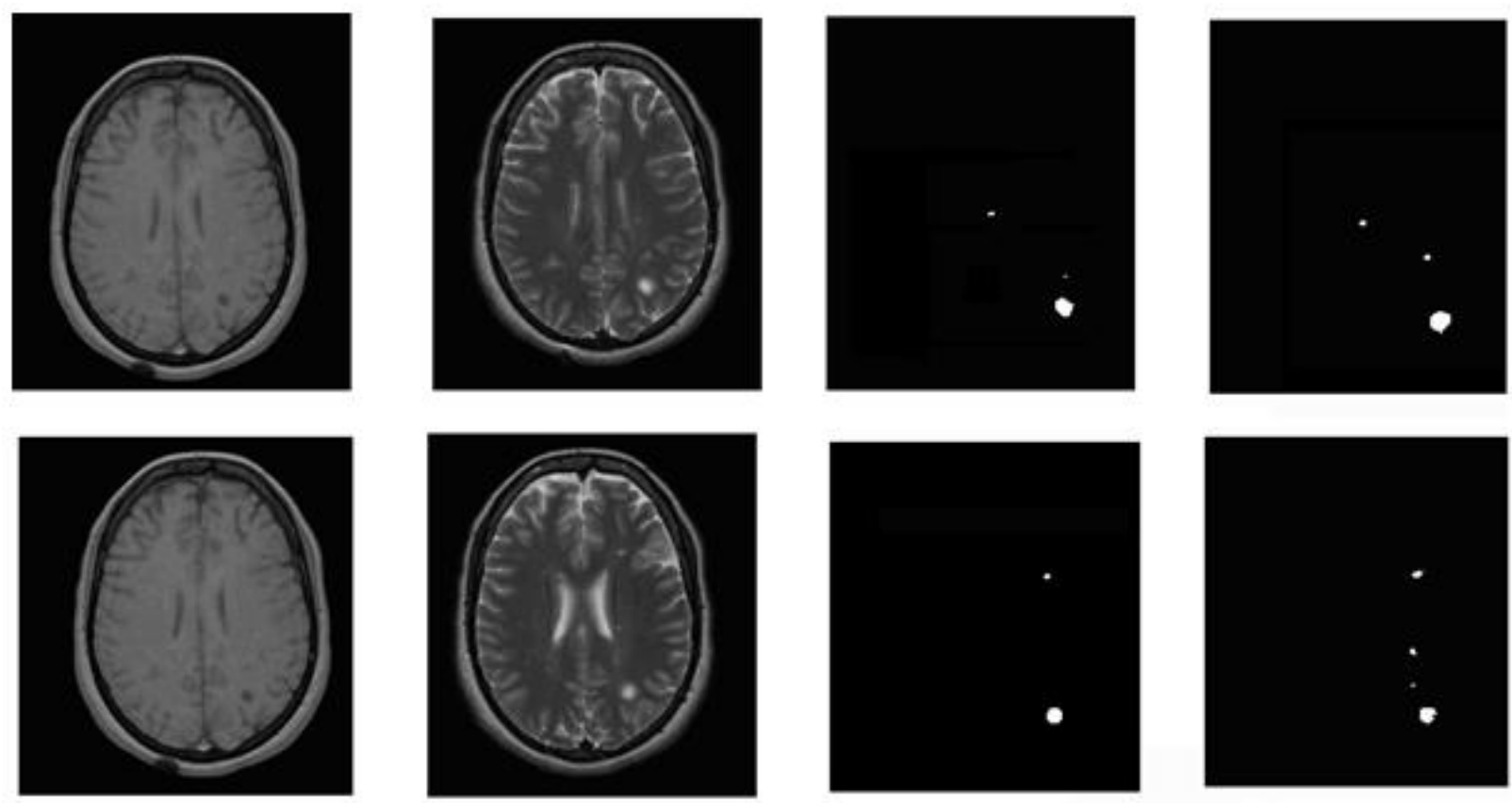

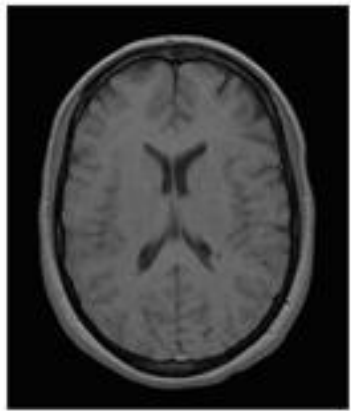

(a)

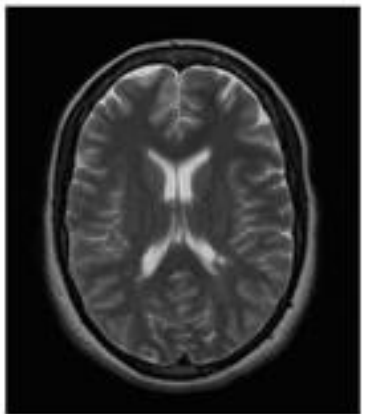

(b)

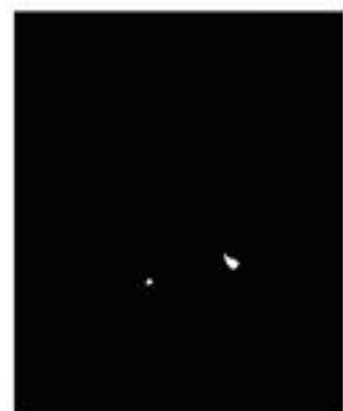

(C)

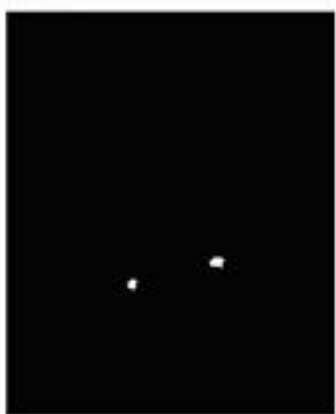

(d)

Figure 6: Segmentation of MS lesions in a real MR images (in three slices from top to down). (a) Original T1-w image. (b) Original T2-w image. (c) Automatic segmentation of MS lesions by SCPFCM method. (d) Manual segmentation of MS lesions.

Table 4 : Comarison of average SI values obtained by several methods in different categories.

\begin{tabular}{l|cccc}
\hline Patient category & K h a y a t i 2 ] & Admiraal[8]Anbeek[3]SCPFCM \\
\hline Small lesion & 0.72 & 0.7 & 0.5 & 0.81 \\
Moderate lesion & 0.75 & 0.75 & 0.75 & 0.82 \\
Large lesion & 0.8 & 0.82 & 0.85 & 0.84 \\
All patients & 0.75 & 0.75 & 0.8 & 0.82 \\
\hline
\end{tabular}

\section{ConClusion}

In this paper, we presented a new method to extract MS lesions based on MPFCM algorithm, tested on two set of simulated and real images. As the results show in simulated images this method works better and more accurate by increasing noise. And also in real images this method is better than the other previous ones in extracting tiny lesions.

\section{ACKNOWLEDGMENT}

We thank the MRI center of Imam Khomeini Hospital in Tehran for providing the MR images. 


\section{REFERENCES}

[1] C. R. Guttmann, R. Kikinis, M. C. Anderson, M. Jakab, S. K. Warfield, R. J. Killiany, H. L. Weiner and F. A. Jolesz, "Quantitative follow-up of patients with multiple sclerosis using MRI: resproducibility,” Journal of Magnetic Resonannce Imaging , 9, pp. 509-518, 1999.

[2] R. Khayati, M. Vafadust, F. Towhidkhah and S. M. Nabavi, “ A novel method for automaticdetermination of different stages of multiple sclerosis lesions in brain MR FLAIR images," Computerized Medical Imaging and Graphics, 32, pp. 124-133, 2008 .

[3] P. Anbeek, K. L. Vincken, M. J. P. van Osch, R. H. C. Bisschops, and J. van der Grond, "Probabilistic segmentation of white matter lesions in MR imaging," NeuroImage 21, pp. 1037-1044, 2004.

[4] D. Lorenzo, S. Prima, S. Morrissey and C. Barillot, "A robust Expectation-Maximization algorithm for Multiple Sclerosis lesion segmentation", in proceeding of MICCAI, 2008.

[5] M. Prastawa and G. Guido, “ Automatic MS Lesion Segmentation by Outlier Detection and Information Theoretic RegionPartitioning,” MIDAS Journal, 2008.

[6] E. Ardizzone, R. Pirrone, O. Gambino and D. Peri, "Two channels fuzzy c-means detection of multiple sclerosis lesions in multispectral MR images,", in International Conference on Image Processing, pp. 345-348, 2002.

[7] F. Admasu, S. Al-Zubi, K. Toennies, N. Bodammer and H Hinrichs, "Segmentation of multiple sclerosis lesions from MR brain images using the principles of fuzzy-connectedness and artificial neuron networks,", in IEEE Proceedings of International Conference on Image Processing, ICIP 2003, pp. 1081-1084, Barcelona, Catalonia, Spain, 2003

[8] F. Admiraal-Behloul, D. M. J. van den Heuvel, H. Olofsen, M. J. P. van Osch, J. van der Grond, M.A. van Buchem and J.H.C. Reiber, "Fully automatic segmentation of white matter hyperintensities in MR images of the elderly," NeuroImage 28 607-617, 2005

[9] R. L Cannon, J. V. Dave and J. C. Bezdek, "Efficient implementation of the fuzzy c-means clustering algoritm", IEEE Trans. on Pattern Analysis and Machine Intelligence, 8(2), pp. 248255, 1986.

[10] R. Krishnapuram and J. M. Keller, "A possibilistic approach to clustering," IEEE Transactions on Fuzzy Systems, 1(2), pp. 98$110,1993$.

[11] N. R. Pal, K. Pal and J. C. Bezdek, "A mixed c-means clustering model," IEEE International Conference on Fuzzy Systems, Vol. 1, pp. 11-21, July 1997.

[12] N. R. Pal, K. Pal, J. M. Keller, J. C. Bezdek, "A possibilistic fuzzy c-means clustering algorithm," IEEE Transactions on Fuzzy Systems, Vol. 13, No. 4, pp. 517-530, Aug. 2005.

[13] H. Khotanlou, J. Atif, O. Collio and I. Bloch,, "3D brain tumor segmentation using fuzzy classification and deformable models", LNCS, Vol. 3849, 312-318, 2005.

[14] Serra, J. (1982). Image analysis and mathematical morphology. Academic Press Inc.,London, UK, 2nd edition.

[15] C. H. Polman, S. C. Reingold, G. Edan, M. Fillippi, H. P. Hartung and L. Kappos, "Diagnostic criteria for MS 2005 revisions to the MC Donald criteria", Ann. Neurol. 58, pp. 840-846, 2005.

[16] C. A. Cocosco, V. Kollokian, R. K. Kwan and A. C. Evans, "Brainweb: Online interface to a 3D MRI simulated brain database", NeuroImage (Proceedings of 3-rd International Conference on Functional Mapping of the Human Brain), Vol. 5 , pp. S425, copenhagen, may 1997. 